\title{
Protección Social y Modelos de Desarrollo en América Latina
}

\section{Latin-American social protection and development models}

\author{
Luz M. Mejía-Ortega y Álvaro Franco-Giraldo
}

Facultad Nacional de Salud Pública, Universidad de Antioquia, Colombia.

luzmmejia@guajiros.udea.edu.co; afranco@guajiros.udea.edu.co

Recibido 2 Enero 2007/Enviado para Modificación 15 Julio 2007/Aceptado 15 Agosto 2007

RESUMEN

Los sistemas de protección social están estrechamente relacionados con los modelos de desarrollo que históricamente se han consolidado en cada nación: Estos de manera implícita o explícita han determinado la política social. El presente artículo trata sobre los modelos de desarrollo y los sistemas de protección social implementados en América Latina, como respuesta a las necesidades de salud y bienestar de la población. Metodológicamente, considera el rol del Estado y los aspectos básicos de la política social como eje de análisis que permite interrelacionar los modelos de desarrollo con los enfoques de protección social en este sub continente. En América Latina, durante el último siglo, los modelos de desarrollo se han fundamentado principalmente en la teoría Keynesiana del Estado de Bienestar (sin llegar a desarrollarse realmente), en industrialización basada en la sustitución de importaciones, en la planificación central, para el caso específico de Cuba, y en la concepción liberal después de los 80. Estos modelos han dado lugar, en cada caso, a sistemas de protección social que han fluctuado entre los enfoques de la seguridad social, el seguro social y la asistencia social, pasando por las redes mínimas de protección social y el manejo social del riesgo. Estos dos últimos impulsados por organismos internacionales en cabeza del Banco Mundial.

Palabras Clave: América Latina, desarrollo humano, bienestar social, seguridad social (fuente: DeCS, BIREME).

\section{ABSTRACT}

Social protection systems are closely related to the development models which each nation has historically consolidated; these have implicitly or explicitly determined social policy. This article deals with Latin-American development models and the social protection systems so implemented as a response to the population's health needs and well-being. It considers the state's role and basic social policy aspects concerning the relationship between development models and approaches to social protection in Latin-America. Latin-American development models during the last century (without being really developed) have been mainly based on Keynesian theory regarding the Welfare State, substitution of imports-based industrialisation, 
central planning (for the specific case of $\mathrm{Cuba}$ ) and liberal approaches since the 1980s. These models have all given rise to social protection systems which have fluctuated between social security approaches, social insurance and social assistance, passing through minimum social protection networks and social handling of risk. The last two have been promoted by international organisms led by the World Bank.

Key Words: Latin-America, social protection, social welfare, social security (source: $\mathrm{MeSH}, \mathrm{NLM})$.

\section{L} a protección social (PS) se ha expresado en programas y sistemas de seguridad social y en políticas sociales para disminuir la pobreza y inequidad, dependiendo de la trayectoria de cada país. En América Latina (AL) durante las últimas décadas se han implementado propuestas y modelos teóricos para construir sistemas de protección social, entre las cuales sobresale la iniciativa del Banco Mundial (BM) sobre "El manejo social del riesgo" encaminada a la prevención, reducción, mitigación y superación del riesgo en grupos poblaciones vulnerables (1).

Diversos análisis han señalado que la PS está determinada por los modelos de desarrollo y por las reformas del Estado y sus posibilidades de financiación. Asimismo, está condicionada por decisiones supranacionales y factores exógenos coyunturales que llevan a implementar determinadas políticas económicas y sociales.

Este artículo pretende develar los nexos de los sistemas de PS con los modelos de desarrollo en AL, que sirva para reorientar la política social y las reformas estructurales del Estado. Parte de reconocer que no existen modelos puros de PS, como bien lo advierten Fleury y Molina (2), en cuanto son abstracciones de ideales que en la práctica presentan diversas características. Se consideran algunos modelos de desarrollo y varias teorías y enfoques de la PS implementados en esta región.

\section{ANTECEDENTES}

Históricamente la PS se ha implementado en los países latinoamericanos bajo esquemas asistenciales, de seguros y seguridad social (3), encontrándose allí "algunos de los más antiguos, fuertes y complejos mecanismos de ingeniería política en el campo de la protección social (...) comparables a los sistemas europeos..." (4). 
La Organización Internacional del Trabajo OIT, la Organización de los Estados Americanos OEA, la Organización Panamericana de la Salud OPS y la Asociación Internacional de la Seguridad Social AISS, la han impulsado a durante el siglo $\mathrm{XX}$, valorando la cooperación nacional e internacional para lograr sus fines, como fue la creación de institutos de seguros sociales, siguiendo el original modelo Bismarckiano (1883-1989).

La PS se renovó en el marco de la seguridad social, después del replanteamiento de los conceptos clásicos de los Seguros Sociales. Este cambio obedeció a las ideas de Beveridge a finales de 1942, posterior a la II Guerra Mundial. Así, la seguridad social, inspirada en principios universales de solidaridad, equidad y justicia social, rescata la responsabilidad estatal y de la sociedad en la PS y se erige como derecho fundamental.

La PS debe entenderse de manera amplia e integral, no sólo limitada al enfoque de riesgo y aseguramiento como actualmente ocurre (1) para ajustarse al rezago económico de los países. Debe incluir todas aquellas variables de orden económico, político y social que garanticen la salvaguardia en eventos adversos que pudieran causar detrimento a la salud, al empleo, al bienestar y a la calidad de vida de las personas, y basarse en criterios de equidad y justicia social.

Así, la OPS y la OIT propusieron la estrategia de la Extensión de la PS para las Américas (EPSS) como una "poderosa herramienta destinada a combatir la exclusión en salud" (5), la cual, aunque se supone ha ocupado espacios en las agendas de los gobiernos y de los organismos internacionales, no ha logrado su carácter prioritario en la política social (6).

\section{LOS MODELOS DE DESARROLLO EN AL}

Los modelos de desarrollo se originan en la forma como una sociedad organiza sus recursos materiales y sus instituciones para buscar el progreso y la satisfacción de necesidades de todos sus miembros, articulado con su historia, su cultura, su producción económica, su tradición jurídico política y sus posibilidades de adelanto científico y tecnológico (7). Considera la extensión de capacidades y complejidades de la sociedad y de los individuos, para ofrecerles mejores posibilidades de satisfacer sus necesidades y aspiraciones (8).

Franco (9), destaca tres modelos que vivió AL en el siglo XX como: a) crecimiento hacia fuera, b) sustitución de importaciones y c) modelo postajuste. Estos modelos estuvieron separados por fechas importantes para el 
desarrollo económico: la crisis económica de 1929 y la crisis de la deuda externa en los años 80.

Otros autores reconocen diferentes modelos de desarrollo a través de la historia. Aquí se describen algunos de ellos (Cuadro1). Aunque en AL no se desarrolló suficientemente el Estado de Bienestar como en los países europeos, se expone porque gran parte del debate actual en la región, parte de la discusión entre Estado de Bienestar y modelo Neoliberal, la cual se retoma en el ensayo. Por la época bienestarista, más bien la política social en los países de AL se debatía entre el modelo basado en la Sustitución de Importaciones y el Desarrollista.

\begin{tabular}{|c|c|c|c|c|c|}
\hline Modelo & $\begin{array}{l}\text { Enfoque } \\
\text { Político }\end{array}$ & $\begin{array}{l}\text { Protección } \\
\text { Social }\end{array}$ & Politica Social & $\begin{array}{c}\text { Experiencias } \\
\text { Pioneras }\end{array}$ & Año \\
\hline $\begin{array}{l}\text { Keynesiano y } \\
\text { Estado de } \\
\text { Bienestar }^{\circ}\end{array}$ & $\begin{array}{l}\text { Estatista } \\
\text { (Estado- } \\
\text { céntrico) }\end{array}$ & $\begin{array}{l}\text { Seguridad } \\
\text { Social } \\
\text { Integral } \\
\text { Universal }\end{array}$ & $\begin{array}{l}\text { Solidaridad y } \\
\text { equidad social }\end{array}$ & $\begin{array}{l}\text { Costa Rica } \\
\text { Uruguay }\end{array}$ & $30-40$ \\
\hline $\begin{array}{l}\text { Basado en } \\
\text { Sustitución de } \\
\text { Importaciones }\end{array}$ & $\begin{array}{l}\text { Estatalista } \\
\text { (Economía } \\
\text { mixta) }\end{array}$ & $\begin{array}{l}\text { Seguros } \\
\text { sociales y } \\
\text { Asistencia } \\
\text { pública }\end{array}$ & $\begin{array}{l}\text { Ampliación del } \\
\text { empleo y gasto } \\
\text { público }\end{array}$ & $\begin{array}{l}\text { Brasil, } \\
\text { México } \\
\text { Argentina } \\
\text { Chile } \\
\text { Colombia }\end{array}$ & 40 s \\
\hline Neoliberal & $\begin{array}{l}\text { Estado de } \\
\text { Derecho } \\
\text { (Mercado- } \\
\text { céntrico) }\end{array}$ & $\begin{array}{l}\text { Redes } \\
\text { Mínimas } \\
\text { de PS } \\
\text { Manejo } \\
\text { Social del } \\
\text { Riesgo }\end{array}$ & $\begin{array}{l}\text { Excluyente } \\
\text { Focalización } \\
\text { del gasto público } \\
\text { Privatización } \\
\text { Descentralización }\end{array}$ & $\begin{array}{l}\text { Bolivia } \\
\text { Perú } \\
\text { Argentina } \\
\text { Chile } \\
\text { Colombia }\end{array}$ & $80 \mathrm{~s}$ \\
\hline $\begin{array}{l}\text { Planificación } \\
\text { Económica } \\
\text { Central }\end{array}$ & $\begin{array}{l}\text { Socialista } \\
\text { (Socio-céntrico) }\end{array}$ & $\begin{array}{l}\text { Politica } \\
\text { Social } \\
\text { Integral }\end{array}$ & $\begin{array}{l}\text { Incluyente } \\
\text { Redistributiva } \\
\text { Énfasis social }\end{array}$ & $\begin{array}{l}\text { Cuba } \\
\text { Nicaragua }\end{array}$ & $\begin{array}{l}60 s \\
70 s\end{array}$ \\
\hline
\end{tabular}

Modelo Keynesiano y Estado de Bienestar

Este modelo, centrado en la teoría general de la ocupación, el interés financiero y el dinero, se ocupa de agregados como ingreso, consumo, ahorro e inversión, más que de la determinación de precios, que hace parte principal de la teoría económica (10). Señala la importancia de las variaciones en el nivel de producción y empleo como movimientos equilibradores de la economía para igualar la inversión y el ahorro, determinándose así el nivel de equilibrio de la renta nacional total y de la producción nacional.

Reconoce que las tasas de ocupación son indicadores de prosperidad en las naciones y que la falta de ingresos compromete seriamente la conservación de la vida y el desarrollo humano (11). 
El Estado de Bienestar surge como la forma de un Estado Social de garantizar estándares mínimos de ingreso, alimentación, salud, vivienda educación y seguridad social a cada ciudadano, sin discriminación alguna, como derecho político "fruto de las luchas sociales que acompañaron los procesos de industrialización y modernización en las sociedades modernas" (12). Fleury y Molina (2) lo reconocen como respuesta al proceso de desarrollo de sociedades capitalistas industrializadas y a sus consecuencias, con el fin de lograr estabilidad y seguridad en un nuevo contexto de solidaridad social.

\section{Modelo basado en la Sustitución de Importaciones}

En su momento fue muy promisorio para la modernización y el progreso de los países de la región y surtió sus efectos sobre altas tasas de crecimiento, mejores oportunidades de empleo y la posibilidad de que muchos pudieran superar sus niveles de pobreza. Sin embargo, el modelo mostró sus primeras señales de postración en la década de los 70 y la región comenzó a perder competitividad en el mundo, generando pérdida de oportunidades para exportar, altos índices de inflación y la consecuente "crisis de la deuda" de los 80 (13), caracterizada por la caída de las tasas de crecimiento, aumento del desempleo, deterioro de salarios, crecimiento de la pobreza y la desigualad.

\section{Modelo Neoliberal}

Se caracteriza por las diversas formas de interacción de los individuos (ciudadanos que automáticamente adquieren la connotación de clientes) en amplios mercados donde los bienes, servicios y valores sociales se convierten en mercancías, con vendedores privados cuyo único interés es la rentabilidad económica bajo el principio de la libre competencia. En este modelo, el consumidor tiene la opción de elegir de acuerdo con su capacidad de compra, pues parte del supuesto de que todos los individuos tienen ingresos suficientes para satisfacer sus necesidades en un mercado que les ofrece una variada gama de bienes y servicios.

Hayek y Friedman, fundan sus principios en la libertad individual y la desigualdad en una franca crítica al Estado benefactor (14), por considerar que "la desigualdad es el motor de la iniciativa personal y la competencia de los individuos en el mercado que corrige todos los males económicos y sociales". El carácter del neoliberalismo es el individualismo en tanto que el individuo liberal es considerado una isla fundamentada en sí misma, centrado en la defensa de sus derechos como individuo a la vida, a la libertad y a la propiedad (11). 
Para sus defensores, "el sistema de precios permite que los individuos cooperen pacíficamente durante breves momentos, mientras que el resto del tiempo cada quien se ocupa de sus propios asuntos" (15), y en consecuencia, según Hayek "en la sociedad moderna y compleja es preciso recurrir al mercado y a la iniciativa individual" (11). En AL, su expansión se dio en los años 80 a partir de la aplicación de reformas de ajuste económico promovidas por el $\mathrm{BM}$ y el Fondo Monetario Internacional, convertidas en recetas para superar el déficit público y estabilizar las economías en esta región (Consenso de Washington) $(16,17,18)$.

\section{Modelo Económico de Planificación Central}

Ha sido propio de la antigua Unión Soviética, Europa Occidental y China y experimentado por iniciativas socialistas en AL (19) como Cuba y Nicaragua, donde el Estado propietario de los recursos disponibles en la economía decide cómo organizar la producción, tipo de industrias y asignación de recursos, así como la combinación de tecnología con los recursos disponibles.

Sus logros en la primera mitad del siglo XX fueron muy significativos tanto en lo ambiental como en la seguridad y el progreso social: aumento de producción e industrialización, garantía de acceso a servicios de educación, salud, vivienda y empleo para grandes poblaciones, y distribución equitativa de ingresos y de servicios sociales. No obstante, por los años 70 comenzó un proceso de estancamiento, obligando al Estado a emprender reformas de economía de mercado (20).

Se han planteado otros modelos alternativos y estrategias de desarrollo tales como el desarrollo sustentable impulsado por la ONU, la teoría del Desarrollo a Escala Humana propuesta por Max Neef y el modelo de transformación productiva con equidad de la CEPAL, pero no logran su aplicación de manera consistente.

\section{ENFOQUES DE PROTECCIÓN SOCIAL}

Aquí se exponen algunos de los enfoques que desde distintas perspectivas se han aplicado en AL durante más de medio siglo. Entre ellos, se identifican tres modelos de PS para la región: seguridad social, seguro social y asistencia social, relacionados por algunos con Estado de Bienestar, Estado LiberalSustitución de Importaciones y modelo Neoliberal, respectivamente (2). 


\section{La Seguridad Social}

Se entiende como el conjunto de medidas previsivas dirigidas a garantizar a los habitantes de un país los medios económicos para lograr condiciones mínimas de comodidad, salud, educación y recreación, así como las providencias contra riesgos inherentes a la vida humana, como desempleo, enfermedad, invalidez parcial o total, vejez, oportunidad de educación y problemas de salud mental (21). Debe pactarse en una política redistributiva (2). En los países europeos incluye además: el pleno empleo, acceso universal a educación, redistribución del ingreso, regulación de relaciones laborales y de condiciones de trabajo, fomento de organizaciones de autoayuda y de negociación entre empresarios y trabajadores. Otras regiones del mundo apenas han alcanzado parcialmente, y más tarde, algunos de estos mecanismos de protección $(22,23)$.

\section{El Seguro Social}

Resulta de la aplicación estratégica de una política de seguridad social al amparo de los principios de solidaridad y universalidad (24) y no como un proceso aislado. Entre sus estrategias está el aseguramiento, definido como "el conjunto de transacciones mediante las cuales el pago de un monto determinado permite recibir otro pago si ocurren ciertos eventos" (25). Por ello, sólo cubre una parte de los riesgos naturales de la vida. No alcanzan a mantener las condiciones mínimas del bienestar, se limitan a cubrir la enfermedad y algunos riesgos económicos, sin garantizar los derechos sociales, pues sólo responden a las demandas surgidas de la industrialización.

\section{La Asistencia Social}

Mecanismo mediante el cual las previsiones financieras corren a cargo del tesoro público. Dirigidas a la porción económicamente más débil de la sociedad, a personas que no pueden contribuir a una caja de previsión, o que, pudiéndolo hacer, todavía no han sido incorporadas a ellas (21). Adicionalmente, otras dos formas de PS aplicados en AL se han basado en la asistencia social: las Redes Mínimas de PS y el Manejo Social del Riesgo (3) propias del post ajuste estructural.

\section{Redes Mínimas de PS (RMPS)}

Surgen como programas asistenciales para los más pobres y vulnerables en respuesta a "la crisis económica y los programas de ajuste estructural que agravaron 
la pobreza y provocaron protestas en algunas ciudades" (22) viéndose amenazada la estrategia neoliberal. Hacia la década del 80, la mayoría de países latinoamericanos entraron en la tónica de las RMPS, con un enfoque descentralizador y participativo, y algunos con énfasis en inversión social. Su finalidad no era más que viabilizar políticamente el ajuste estructural (3) para mejorar la eficiencia de algunos ministerios.

De acuerdo con Mesa Lago (22), en AL surgieron varios tipos de RMPS tales como los programas de acción social (PAS), en Costa Rica y México; los Fondos de Emergencia Social (FES) en Argentina, Bolivia, Colombia, El Salvador, Perú y Uruguay; los Fondos de Inversión Social (FIS) en Bolivia y Chile. Su financiación ha sido con recursos del Banco Interamericano de Desarrollo (BID), del BM y de la Agencia Interamericana de Desarrollo (AID), entre otros.

Tales Redes, caracterizadas por su ánimo mitigador y paliativo, no logran la protección amplia de la seguridad social integral (26) ni alcanzan a enfrentar todas las dimensiones del riesgo. Según Ana Sojo (25), surgen como una propuesta global de política social con una combinación público-privada, en la cual las responsabilidades estatales en materia de bienestar social se circunscriben al combate contra la pobreza y el aseguramiento contra los riesgos adquiere una dimensión individual, desvirtuando el carácter solidario de la seguridad social.

\section{Manejo Social del Riesgo}

Esta propuesta del BM define la PS como "intervenciones públicas para asistir a personas, hogares y comunidades a mejorar su manejo del riesgo y proporcionar apoyo a quienes se encuentran en la extrema pobreza" (1). Tal estrategia debe dirigirse especialmente a los menos favorecidos, a los que Guerra de Macedo llama "los sin ingreso, sin vivienda, sin educación, sin salud, (...) y especialmente los sin poder" (27).

Este enfoque se basa en que todas las personas, hogares y comunidades son susceptibles de múltiples riesgos naturales y antrópicos altamente relacionados con la pobreza. El Banco, identifica como actores involucrados a individuos, comunidades, ONG gobiernos y organismos internacionales (1), pero no a los grandes poseedores de riqueza, lo cual deja sin opciones de lograr los propósitos de superación de la pobreza, mejoramiento de las condiciones de vida y equidad. 
Algunas experiencias por países

La PS en AL ha estado condicionada de manera diversa no sólo por el modelo de desarrollo sino también por el régimen político, los aspectos financieros y las falencias administrativas que han afectado los mecanismos de seguridad social. Esto se ilustra con los casos de Brasil y Colombia donde el determinante principal es la búsqueda de legitimidad política. La PS hace parte de los sistemas de mediación y articulación entre lo político y lo económico (28). De ahí la diversidad entre países y las tipificaciones de PS.

\begin{tabular}{|c|c|c|c|c|}
\hline Clasificación & Paises & Origen & Configuración & Periodo \\
\hline Pioneros & $\begin{array}{l}\text { Argentina } \\
\text { Brasil } \\
\text { Chile } \\
\text { Uruguay }\end{array}$ & Estados populistas & $\begin{array}{l}\text { Gradual } \\
\text { estratificada }\end{array}$ & $1920-1930$ \\
\hline Intermedios & $\begin{array}{l}\text { México } \\
\text { Costa Rica } \\
\text { Panamá, } \\
\text { Países } \\
\text { Andinos }\end{array}$ & $\begin{array}{l}\text { Transición de } \\
\text { crecimiento primario } \\
\text { exportador a } \\
\text { sustitución de } \\
\text { importaciones }\end{array}$ & $\begin{array}{l}\text { Heterogéneo, } \\
\text { Cobertura urbana }\end{array}$ & $1940-1950$ \\
\hline Tardios & $\begin{array}{l}\text { Centro- } \\
\text { Americanos } \\
\text { Caribe, } \\
\text { Anglófonos }\end{array}$ & $\begin{array}{l}\text { Formas pre- } \\
\text { capitalistas }\end{array}$ & $\begin{array}{l}\text { Embrionaria, } \\
\text { incipiente } \\
\text { Unificados y } \\
\text { uniformes }\end{array}$ & $1960-1970$ \\
\hline Post-reforma & $\begin{array}{l}\text { Chile } \\
\text { Bolivia } \\
\text { México } \\
\text { Perú } \\
\text { Colombia } \\
\text { Argentina } \\
\text { Uruguay } \\
\text { Cusla Rica } \\
\text { Brasil }\end{array}$ & $\begin{array}{l}\text { Neoliberal } \\
\text { Neoliberal }\end{array}$ & $\begin{array}{l}\text { Privado sustitutivo } \\
\text { Paralelo (público o } \\
\text { privado) } \\
\text { Mixto (público y } \\
\text { privado) }\end{array}$ & $1990-2000$ \\
\hline
\end{tabular}

Mesa Lago (29) clasifica los países de la región en pioneros, intermedios y tardíos (Cuadro 2) de acuerdo con el momento en que se establece la seguridad social y su desarrollo posterior (siglo XX, hasta los años 80). Diferencia mecanismos financieros, de gestión y de equidad social dentro de de la misma clasificación, según antigüedad de programas, cobertura poblacional, carga salarial y económica, equilibrio financiero y aspectos demográficos, etc.

Esta variabilidad trae distorsiones, de orden micro y macroeconómicas, en los modelos y tipificaciones descritas en la PS dependiendo de las relaciones del Estado con la sociedad en cada caso, y de su conflictividad interna. Su desarrollo tampoco fue armónico en los países y hacia los años 80 el 90\% del 
gasto se concentraba en programas de pensiones, enfermedad-maternidad y el $10 \%$ restante en riesgos profesionales, desempleo y asignaciones familiares.

En los últimos 30 años, AL ha entrado en procesos de reforma variando los mecanismos de PS en pensiones (Cuadro 2), salud y otros servicios sociales (30) pero a pesar de las especificidades de los distintos modelos se da una tendencia única de reforma neoliberal.

Otros análisis (31) demuestran diferencias en las configuraciones de sistemas de salud en AL, dependientes del modelo de Estado y del modelo de desarrollo: así, se da el sistema nacional de salud de Cuba, donde prevalece un modelo de desarrollo basado en la planificación centralizada; en contraste, sistemas basados en el aseguramiento individual en Colombia y República Dominicana, países en los cuales se impone el Modelo Neoliberal; y como casos intermedios, los sistemas segmentados en Bolivia, Ecuador y Venezuela, países que arrastran el rezago de modelos desarrollistas de la época previa a la reforma del Estado.

\section{DISCUSIÓN}

En AL, de acuerdo con Franco, "cada modelo de desarrollo tiene implícito o explícito una concepción de la cuestión social" (9). Estos modelos han sido considerados como simplificaciones estilizadas de los rasgos que caracterizan las diferentes etapas del desarrollo y no logran representar totalmente la situación social, ni responder a la conflictividad interna de cada país. Cada modelo de desarrollo se acompaña de su propia política social y a su vez condiciona el sistema de PS imperante en cada país y aunque históricamente se dieron las singularidades descritas atrás, también se fueron configurando generalidades (modelos o tipificaciones ideales de PS).

En el análisis de AL en el siglo XX (2,9), la etapa previa a la crisis del capitalismo del 29, corresponde al Estado Liberal clásico; la de sustitución de importaciones, al Estado social, planificador e interventor; y la etapa posterior al ajuste, corresponde al Estado Neoliberal.

El primero, se basa en un modelo de desarrollo de mercado externo y su política social se basa en la iniciativa privada, en el auto proteccionismo como forma de PS y en prácticas privadas de mutualismo. El segundo, basado en el mercado interno, fundamenta la política social en la protección de los trabajadores asalariados y en el seguro social que cubre la relación capital trabajo. Finalmente, el Estado Neoliberal, vuelve con la apertura económica y 
la confianza en el mercado externo y auspicia una política neosocial atomizada y selectiva, manteniendo su énfasis en la generación de capital humano. En este último, el modelo de PS se basa en las Redes Mínimas de PS (RMPS), en la focalización y en los seguros privados.

Otros consideran que las políticas sociales son "expresiones fragmentadas de las relaciones de clase" (32), denotando su aparición en áreas separadas del Estado, aparentemente autónomas: política económica, política laboral y política social. En este sentido, Fleury (4) sostiene que el ciudadano es sujeto principal en el modelo de seguridad social integral, donde las prestaciones universales son un verdadero derecho ciudadano (ciudadanía universal). No obstante, en AL nunca lo fue y más bien aparece un nuevo sujeto: el trabajador ligado al contrato de trabajo (ciudadanía regulada), lo que efectivamente corresponde al aseguramiento (seguros sociales) basado en la relación capital-trabajo y en la contribución (PS). De otro lado, el asistencialismo, mantiene en los pobres un nuevo sujeto legítimo y único sobre el cual recae la política: el pobre y el desempleado quienes, mediante la focalización de la política social, se convierten en "sujetos del asistencialismo" (ciudadanía invertida).

\section{CONCLUSIONES}

En general, la PS está condicionada por el modelo de desarrollo imperante y por la forma como éste se articula con las estructuras del Estado en la mediación Estado-Sociedad. Surgen, así, modelos ideales de seguridad social, aseguramiento y asistencia social. Sin embargo, la experiencia latinoamericana muestra un mapa abigarrado de configuraciones de PS condicionadas por otros determinantes históricos y políticos los cuales moldean el modelo de desarrollo.

Idealmente, los modelos de desarrollo deberían orientarse a superar los problemas de pobreza, desempleo y deuda social acumulada con iniciativas propias de PS y no basadas en formulaciones externas que no reconocen las diferencias económicas, políticas y sociales de los países •

\section{REFERENCIAS}

1. Holzmann R, Jorgensen S. Manejo Social del Riesgo: Un nuevo marco conceptual para la Protección Social y más allá. [Internet] Disponible en: http:// info.worldbank.org/etools/docs/library/79020/Fall\%202001/elearning/fall2001/ readings/pdfpapers/manejo.pdf. Consultado julio de 2007. 
2. Fleury S, Molina C. Modelos de Protección social. En: Banco Interamericano de Desarrollo, Instituto Interamericano para el Desarrollo Social (INDES). Diseñoy gerencia de políticas y programas sociales. Washington: INDES; 2002. p. 3-6.

3. Franco A. El Auge de la Protección Social. Revista Facultad Nacional de Salud Pública 2005; 23(1):135-47.

4. Fleury S. Estado sin ciudadanos. Seguridad Social en América Latina. Buenos Aires: Lugar Editorial SA; 1997. p. 212.

5. OPS-OIT. Ampliación de la protección social en salud: una iniciativa conjunta de la Organización Panamericana de la Salud y de la Organización Internacional del Trabajo. Washington DC; 2002.

6. OPS, OMS, ASDI. Exclusión en Salud en países de América Latina y el Caribe. Serie No 1 Extensión dela Protección Social en Salud. Washington, D.C.: La Organización; 2003.

7. Cardona A. Economía, salud-enfermedad y modelos de desarrollo. En: Carlos E, Rosa S, Gloria G (compiladores). Cultura y Salud en la Construcción de las Américas. Bogotá: Instituto Colombiano de Antropología y Cultura; 1993. p. 156

8. Agudelo C. Desarrollo y Salud. Revista de Salud Pública 1999; 1(1): 18.

9. Franco R. Los paradigmas de la política social en América Latina. En: Alcaldía de Medellín (compiladora). Políticas Públicas y Gestión Social: Una Mirada desde la Gerencia Social. Medellín: Municipio de Medellín; 1997.p. 19-34

10. Schwartz P. El comercio internacional en la historia del pensamiento económico. IUDEM Documento de Trabajo 2001-3. p 35-40 [Internet]: Disponible en: http:/ /www.ucm.es/info/iudem/2001-3.pdf Consultado junio de 2006.

11. Musgrove P. Relaciones entre la salud y el desarrollo. Boletín de la Oficina Sanitaria Panamericana 1993; 114(2):118-9

12. Sarmiento L. La política social en dos escenarios futuros. En: Rojas MC, Delgado A (compiladoras). Política social, desafíos y utopías. Memorias del Seminario internacional nuevas tendencias en política social. Bogotá: Pontificia Universidad Javeriana; 1997. p. 289-313

13. Rey de Marulanda N. América Latina: pobreza y desigualad durante 50 años de reformas económicas y sociales. Washington: Banco Interamericano de Desarrollo/ Instituto Interamericano para el Desarrollo Social; 2000. p. 6-7

14. Laurell AC. La política social en el proyecto neoliberal. Necesidades económicas y realidades sociopolíticas. Cuadernos Médico Sociales 1992; 60: p 3-8

15. Friedman M. Libertad de elegir. Barcelona: Ediciones Grijalbo; 1980. p. 61.

16. CLAD - Centro Latinoamericano de Administración para el Desarrollo-. Una Nueva Gestión Pública para América Latina. Documento preparado por el Consejo Científico del CLAD [Internet] Disponible en: http://unpan1.un.org/intradoc/ groups/public/documents/CLAD/UNPAN000161 Consultado 1/05/2004. 
17. Williamson J. Latin American Adjustment: how much has happened? Washington DC: Institute for International Economics; 1990. p. 738

18. Gentili P. El Consenso de Washington yla Crisis de la Educación en América Latina. En: Álvarez F, Santesmases AJ, Muguerza J, Pastor J, Rendueles G, Varela J (compiladores). Neoliberalismo Vs. Democracia. Madrid: La Piqueta; 1998.p.122

19. Cardona J. El concepto de salud enfermedad y salud pública según los diferentes modos deproducción. Revista centroamericana de administración pública 1987; (12): 103-136

20. Banco Mundial. Informe sobre el desarrollo mundial 1996. De la planificación centralizada a la economía de mercado. Washington, DC: 1996. p. 133-148

21. Zúñiga M. Seguridad Social y su Historia. Caracas: Universidad Central de Venezuela; 1963.p.19

22. Mesa-Lago C. Desarrollo social, Reforma del Estado y de la seguridad social al umbral del siglo XXI. Serie Políticas Sociales Nº 36 CEPAL/ELAC. Santiago de Chile: 2000

23. Martínez D, Vega ML. La globalización gobernada. Estado, sociedad y mercado en el siglo XXI. Madrid: Editorial Tecnos (Grupo Anaya); 2001

24. Titelman D, UthoffA. El papel del aseguramiento en la protección social. Revista de la CEPAL. 2003; 81: 103-122

25. Sojo A. Vulnerabilidad social, aseguramiento y diversificación de riesgos en América Latina yel Caribe. Revista CEPAL 2003; 80: 121-140

26. BeveridgeW. Las bases de la seguridad social. México: Fondo de Cultura Económica; 1987

27. Guerra C. La extensión de la protección social en salud en el nuevo Estado latinoamericano. Revista del CLAP. (Caracas) 2000; (19): 69

28. Le Bonniec Y. Sistemas de protección social comparados: Colombia, Brasil y México. Revista Salud Pública 2002; 4(3): 203-239.

29. Mesa-Lago C. Desarrollo de la Seguridad Social en América Latina. CEPAL. 1985.

30. Franco A. Sistemas Previsionales, Salud y Reformas en América Latina. Revista Salud Pública. 2001; 3(3): 223-244.

31. Gómez-Camelo D. Análisis comparativos de los sistemas de salud de la región Andina y el Caribe. Revista Salud Pública 2005; 7(3):305-316.

32. Grassi E, Hintze S, Neufeld MR. Políticas Sociales y Sujetos de Derecho. Una Comparación entre las políticas educativas a nivel primario, el subsector de obras sociales y las políticas de asistencia alimentaria. Cuadernos Médico Sociales 1995; 72: 62 (62-76). 